\title{
Interval-valued Fuzzy Ideal of N(2,2,0) Algebra
}

\author{
Fengxiao Wang \\ College of Mathematics and Statistics, Kashigar University, Kashi, 844000, China
}

\begin{abstract}
The purpose of this paper is to define the notion of an interval-valued fuzzy ideal of $N(2,2,0)$ algebra. Necessary and sufficient conditions for an i-v fuzzy set to be i-v fuzzy ideals are stated. It is proved that the intersection and direct product in $\mathbf{N}$ $(2,2,0)$ algebra of $i-v$ fuzzy ideal are also i-v fuzzy ideal. The results enriched fuzzy theory in $N(2,2,0)$ algebra.
\end{abstract}

Keywords-N(2,2,0) algebra; interval-valued fuzzy set; intervalvalued fuzzy ideal; intersection; direct product

\section{INTRODUCTION}

The $\mathrm{N}(2,2,0)$ algebra is an algebra system with two dual semigroups. The related properties of $\mathrm{N}(2,2,0)$ algebra have been discussed in literatures[1-8]. The concept of a fuzzy set, which was introduced by L.A.Zadeh [9], provides a natural framework for generalizing many of the concepts of general mathematics. Since then these fuzzy ideal and fuzzy subalgebra have been applied to other algebraic structures such as semigroups, groups, rings, $\mathrm{BCI} / \mathrm{BCH}$-algebra, etc. In [10], Zadeh made an extension of the concept of a fuzzy set by an interval-valued fuzzy set. This interval-valued fuzzy set is referred to as an i-v fuzzy set. In [10], Zadeh also constructed a method of approximate inference using i-v fuzzy sets. Biswas defined interval-valued fuzzy subgroups and investigated some elementary properties. In this paper, using the notion of interval-valued fuzzy set, the concept of $\mathrm{i}-\mathrm{v}$ fuzzy ideal is introduced of $\mathrm{N}(2,2,0)$ algebra. Necessary and sufficient conditions for i-v fuzzy set to be i-v fuzzy ideals are stated. It is proved that the intersection and direct product in $\mathrm{N}(2,2,0)$ algebra of i-v fuzzy ideal are also i-v fuzzy ideal.

\section{PRELIMINARIES}

In this section, we include some elementary aspects that are necessary for this paper.

Definition 2.1 An algebra $(S, *, \Delta, 0)$ is called a $N(2,2,0)$ algebra if it satisfies the following conditions:

(1) $x^{*}(y \Delta z)=z *(x * y)$

(2) $(x \Delta y) * z=y^{*}\left(x^{*} z\right)$

(3) $0 * x=x$

for all $x, y, z \in S$

Any $N(2,2,0)$ algebra $(S, *, \Delta, 0)$ satisfies the following axioms: for all $x, y, z \in S$,
(1) $x^{*} y=y \Delta x$

(2) $\left(x^{*} y\right)^{*} z=x^{*}\left(y^{*} z\right),(x \Delta y) \Delta z=x \Delta(y \Delta z)$

If $(S, *, \Delta, 0)$ is $\mathrm{N}(2,2,0)$ algebra, then $\left(S,{ }^{*}\right)$ and $(S, \Delta)$ are dual semigroup.

Definition 2.2 A subset $I$ of $\mathrm{N}(2,2,0)$ algebra $(S, *, \Delta, 0)$ is called ideal of $\mathrm{S}$ if it satisfies

(1) $0 \in I$, (2) $\forall x, y \in S, x^{*} y \in I, y \in I \Rightarrow x \in I$.

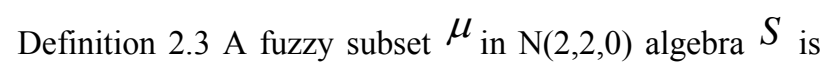
said to be a fuzzy ideal of $\mathrm{S}$ if it satisfies, for all $x, y \in S$

(1) $\mu(0) \geq \mu(x)$, (2) $\mu(x) \geq \mu(x * y) \wedge \mu(y)$.

An interval-valued fuzzy set (briefly, i-v fuzzy set) A defined on $\mathrm{S}$ is given by $A=\left\{x,\left[\mu_{A}^{L}(x), \mu_{A}^{U}(x)\right]\right\}, \forall x \in S$ (briefly, denoted by $A=\left[\mu_{A}^{L}, \mu_{A}^{U}\right]$ ), where $\mu_{A \text { and }}^{L} \mu_{A}^{U}$ are two fuzzy sets in $S$ such that $\mu_{A}^{L}(x) \leq \mu_{A}^{U}(x)$ for all $x \in S$ Let $\bar{\mu}_{A}=\left[\mu_{A}^{L}, \mu_{A}^{U}\right]_{\text {and }} D[0,1]_{\text {denotes the family of all }}$ closed subintervals of $[0,1]$. Thus $\bar{\mu}_{A}(x) \in D[0,1]$ and therefore the $\mathrm{i}-\mathrm{v}$ fuzzy set $\mathrm{A}$ is given by $A=\left\{x, \bar{\mu}_{A}(x)\right\}, \forall x \in S, \bar{\mu}_{A}: S \rightarrow D[0,1]$.

Now let us define what is known as refined minimum (briefly, $\gamma_{\min }$ ) of two elements in $D[0,1]$. Consider two elements $D_{1}=\left[a_{1}, b_{1}\right]$ and $D_{2}=\left[a_{2}, b_{2}\right] \in D[0,1]$.Then $\gamma_{\text {min }}\left(D_{1}, D_{2}\right)=\left[\min \left\{a_{1}, a_{2}\right\}, \min \left\{b_{1}, b_{2}\right\}\right] ; D_{1} \geq D_{2}$ if and only if $a_{1} \geq a_{2}, b_{1} \geq b_{2}$.

III. INTERVAL-VALUED FUZZY IDEAL OF N(2,2,0) ALGEBRA

In what follows, let $\mathrm{S}$ denote a $\mathrm{N}(2,2,0)$ algebra unless otherwise specified.

Definition 3.1 An i-v fuzzy set A in S is called an interval -valued fuzzy ideal (i-v fuzzy ideal) of $S$ if it satisfies the following conditions: 
( I ) $\bar{\mu}_{A}(0) \geq \bar{\mu}_{A}(x)$

(II) $\bar{\mu}_{A}(x) \geq \gamma_{\min }\left(\bar{\mu}_{A}\left(x^{*} y\right), \bar{\mu}_{A}(y)\right)$

or $\bar{\mu}_{A}(x) \geq \gamma_{\min }\left(\bar{\mu}_{A}(y \Delta x), \bar{\mu}_{A}(y)\right)$

for all $x, y \in S$.

Theorem 3.2 An i-v fuzzy set $A=\left[\mu_{A}^{L}, \mu_{A}^{U}\right]_{\text {in } \mathrm{S} \text { is an i-v }}$ fuzzy ideal of $\mathrm{S}$ if and only if $\mu_{A}^{L}$ and $\mu_{A}^{U}$ are fuzzy ideals of S.

Proof Suppose that $\mu_{A}^{L}$ and $\mu_{A}^{U}$ are fuzzy ideals of S. Let $x, y \in S$

Then $\mu_{A}^{L}(0) \geq \mu_{A}^{L}(x) \quad \mu_{A}^{L}(x) \geq \mu_{A}^{L}\left(x^{*} y\right) \wedge \mu_{A}^{L}(y)$, $\mu_{A}^{U}(0) \geq \mu_{A}^{U}(x), \mu_{A}^{U}(x) \geq \mu_{A}^{U}\left(x^{*} y\right) \wedge \mu_{A}^{U}(y)$.

It follows that

$$
\begin{aligned}
& \bar{\mu}_{A}(0)=\left[\mu_{A}^{L}(0), \mu_{A}^{U}(0)\right] \geq\left[\mu_{A}^{L}(x), \mu_{A}^{U}(x)\right]=\bar{\mu}_{A}(x), \\
& \bar{\mu}_{A}(x)=\left[\mu_{A}^{L}(x), \mu_{A}^{U}(x)\right] \\
\geq & {\left[\mu_{A}^{L}\left(x^{*} y\right) \wedge \mu_{A}^{L}(y), \mu_{A}^{U}\left(x^{*} y\right) \wedge \mu_{A}^{U}(y)\right] } \\
= & \gamma_{\min }\left(\left[\mu_{A}^{L}\left(x^{*} y\right), \mu_{A}^{U}\left(x^{*} y\right)\right],\left[\mu_{A}^{L}(y), \mu_{A}^{U}(y)\right]\right) \\
= & \gamma_{\min }\left(\bar{\mu}_{A}\left(x^{*} y\right), \bar{\mu}_{A}(y)\right)
\end{aligned}
$$

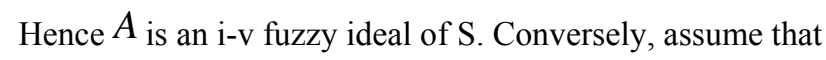
$A$ is an i-v fuzzy ideal of $\mathrm{S}$. Then for any $x, y \in S$, we have $\bar{\mu}_{A}(0) \geq \bar{\mu}_{A}(x)$, that is

$$
\begin{aligned}
& {\left[\mu_{A}^{L}(0), \mu_{A}^{U}(0)\right] \geq\left[\mu_{A}^{L}(x), \mu_{A}^{U}(x)\right]} \\
& \text { Therefore } \mu_{A}^{L}(0) \geq \mu_{A}^{L}(x), \mu_{A}^{U}(0) \geq \mu_{A}^{U}(x) \text {. Since }
\end{aligned}
$$$$
\bar{\mu}_{A}(x) \geq \gamma_{\min }\left(\bar{\mu}_{A}\left(x^{*} y\right), \bar{\mu}_{A}(y)\right)
$$$$
=\gamma_{\min }\left(\left[\mu_{A}^{L}\left(x^{*} y\right), \mu_{A}^{U}\left(x^{*} y\right)\right],\left[\mu_{A}^{L}(y), \mu_{A}^{U}(y)\right]\right)
$$$$
=\left[\mu_{A}^{L}\left(x^{*} y\right) \wedge \mu_{A}^{L}(y), \mu_{A}^{U}\left(x^{*} y\right) \wedge \mu_{A}^{U}(y)\right]
$$$$
\text { Hence } \mu_{A}^{L}(x) \geq \mu_{A}^{L}\left(x^{*} y\right) \wedge \mu_{A}^{L}(y),
$$$$
\mu_{A}^{U}(x) \geq \mu_{A}^{U}\left(x^{*} y\right) \wedge \mu_{A}^{U}(y)
$$

It follows that $\mu_{A}^{L}$ and $\mu_{A}^{U}$ are fuzzy ideals of S.

Theorem 3.3 Let A is an i-v fuzzy set in S. Then A is an i$v$ fuzzy ideal of $S$ if and only if the nonempty set

$$
U\left(A,\left[\delta_{1}, \delta_{2}\right]\right)=\left\{x \in S \mid \bar{\mu}_{A}(x) \geq\left[\delta_{1}, \delta_{2}\right]\right\}
$$

is an ideal of S for every $\left[\delta_{1}, \delta_{2}\right] \in D[0,1]$.

Proof Assume that $\mathrm{A}$ is an $\mathrm{i}-\mathrm{v}$ fuzzy ideal of $\mathrm{S}$ and let $\left[\delta_{1}, \delta_{2}\right] \in D[0,1]$ be such that $x \in U\left(A,\left[\delta_{1}, \delta_{2}\right]\right)$ Then $\bar{\mu}_{A}(0) \geq \bar{\mu}_{A}(x) \geq\left[\delta_{1}, \delta_{2}\right]$. Thus $0 \in U\left(A,\left[\delta_{1}, \delta_{2}\right]\right)$.

$$
\begin{aligned}
& \text { If } x^{*} y \in U\left(A,\left[\delta_{1}, \delta_{2}\right]\right) \text { and } y \in U\left(A,\left[\delta_{1}, \delta_{2}\right]\right) \text {, then } \\
& \bar{\mu}_{A}(x * y) \geq\left[\delta_{1}, \delta_{2}\right], \bar{\mu}_{A}(y) \geq\left[\delta_{1}, \delta_{2}\right]
\end{aligned}
$$

Since A is an i-v fuzzy ideal, therefore $\bar{\mu}_{A}(x) \geq \gamma_{\min }\left(\bar{\mu}_{A}\left(x^{*} y\right), \bar{\mu}_{A}(y)\right)$

$\geq \gamma_{\min }\left(\left[\delta_{1}, \delta_{2}\right],\left[\delta_{1}, \delta_{2}\right]\right)=\left[\delta_{1}, \delta_{2}\right]$ That is to say $x \in U\left(A,\left[\delta_{1}, \delta_{2}\right]\right)$, hence $U\left(A,\left[\delta_{1}, \delta_{2}\right]\right)$ is an ideal of $\mathrm{S}$. Conversely, assume that nonempty set $U\left(A,\left[\delta_{1}, \delta_{2}\right]\right)$ is an ideal of $\mathrm{S}$ for every $\left[\delta_{1}, \delta_{2}\right] \in D[0,1]$. Suppose there exists $a \in S$ such that $\left[c_{1}, c_{2}\right]=\bar{\mu}_{A}(0)<\bar{\mu}_{A}(a)=\left[a_{1}, a_{2}\right]$, taking $\left[b_{1}, b_{2}\right]=\left(\left[c_{1}, c_{2}\right]+\left[a_{1}, a_{2}\right]\right) / 2$, then $0 \notin U\left(A,\left[b_{1}, b_{2}\right]\right)$. This is a contradiction since $U\left(A,\left[b_{1}, b_{2}\right]\right)$ is ideal of $\mathrm{S}$, therefore $\bar{\mu}_{A}(0) \geq \bar{\mu}_{A}(x)$ for all $x \in S$.Suppose there exist $x_{0}, y_{0} \in S$ $\bar{\mu}_{A}\left(x_{0}\right)<\gamma_{\min }\left(\bar{\mu}_{A}\left(x_{0} * y_{0}\right), \bar{\mu}_{A}\left(y_{0}\right)\right)$ that $\bar{\mu}_{A}\left(x_{0}\right)=\left[\delta_{1}, \delta_{2}\right] \quad, \quad \bar{\mu}_{A}\left(y_{0}\right)=\left[r_{3}, r_{4}\right]$ $\bar{\mu}_{A}\left(x_{0} * y_{0}\right)=\left[r_{1}, r_{2}\right]$ Then

$$
\left[\delta_{1}, \delta_{2}\right]<\gamma_{\min }\left(\left[r_{1}, r_{2}\right],\left[r_{3}, r_{4}\right]\right)=\left[\min \left(r_{1}, r_{3}\right), \min \left(r_{2}, r_{4}\right)\right]
$$
Hence $\delta_{1}<\min \left(r_{1}, r_{3}\right)$ and $\delta_{2}<\min \left(r_{2}, r_{4}\right)$.Taking

$$
\left[\lambda_{1}, \lambda_{2}\right]=\frac{1}{2}\left(\bar{\mu}_{A}\left(x_{0}\right)+\gamma_{\min }\left(\bar{\mu}_{A}\left(x_{0} * y_{0}\right), \bar{\mu}_{A}\left(y_{0}\right)\right),\right.
$$

we obtain

$$
\begin{aligned}
& {\left[\lambda_{1}, \lambda_{2}\right]=\frac{1}{2}\left(\left[\delta_{1}, \delta_{2}\right]+\left[\min \left(r_{1}, r_{3}\right), \min \left(r_{2}, r_{4}\right)\right]\right)} \\
& =\left[\frac{1}{2}\left(\delta_{1}+\min \left(r_{1}, r_{3}\right)\right), \frac{1}{2}\left(\delta_{2}+\min \left(r_{2}, r_{4}\right)\right)\right]
\end{aligned}
$$

It follows that 


$$
\min \left(r_{1}, r_{3}\right)>\lambda_{1}=\frac{1}{2}\left(\delta_{1}+\min \left(r_{1}, r_{3}\right)\right)>\delta_{1}
$$

$\min \left(r_{2}, r_{4}\right)>\lambda_{2}=\frac{1}{2}\left(\delta_{2}+\min \left(r_{2}, r_{4}\right)\right)>\delta_{2}$

So that

$$
\left[\min \left(r_{1}, r_{3}\right), \min \left(r_{2}, r_{4}\right)\right]>\left[\lambda_{1}, \lambda_{2}\right]>\left[\delta_{1}, \delta_{2}\right]=\bar{\mu}_{A}\left(x_{0}\right)
$$

Therefore $x_{0} \notin U\left(A,\left[\lambda_{1}, \lambda_{2}\right]\right)$. On the other hand,

$\bar{\mu}_{A}\left(x_{0}^{*} y_{0}\right) \geq\left[\min \left(r_{1}, r_{3}\right), \min \left(r_{2}, r_{4}\right)\right]>\left[\lambda_{1}, \lambda_{2}\right]$ $\bar{\mu}_{A}\left(y_{0}\right)=\left[r_{3}, r_{4}\right] \geq\left[\min \left(r_{1}, r_{3}\right), \min \left(r_{2}, r_{4}\right)\right]>\left[\lambda_{1}, \lambda_{2}\right]$.

Thus $x_{0}^{*} y_{0} \in U\left(A,\left[\lambda_{1}, \lambda_{2}\right]\right)$ and $y_{0} \in U\left(A,\left[\lambda_{1}, \lambda_{2}\right]\right)$. It contradicts that $U\left(A,\left[\lambda_{1}, \lambda_{2}\right]\right)$ is ideal of $\mathrm{S}$. Hence for all $x, y \in S$,

$$
\bar{\mu}_{A}(x) \geq \gamma_{\min }\left(\bar{\mu}_{A}\left(x^{*} y\right), \bar{\mu}_{A}(y)\right) .
$$

This completes the proof.

Theorem 3.4 Every ideal of S can be realized as an i-v level set of i-v fuzzy ideal of S.

Proof Let $\mathrm{Y}$ be an ideal of S and Let A be an i-v fuzzy set on $\mathrm{S}$ defined by

$$
\bar{\mu}_{A}(x)=\left\{\begin{array}{cc}
{\left[\alpha_{1}, \alpha_{2}\right]} & x \in Y \\
{[0,0]} & x \notin Y
\end{array}\right.
$$

where $\alpha_{1}, \alpha_{2} \in[0,1]_{\text {and }} \alpha_{1}<\alpha_{2}$.

It is clear that $U\left(A,\left[\alpha_{1}, \alpha_{2}\right]\right)=Y$. We show that $\mathrm{A}$ be an i-v fuzzy ideal of S. Let $x, y \in S$.

If $x^{*} y \in Y$ and $y \in Y$, then $x \in Y$, this shows that

$$
\bar{\mu}_{A}(x)=\bar{\mu}_{A}(y)=\bar{\mu}_{A}(x * y)=\left[\alpha_{1}, \alpha_{2}\right] \text {. }
$$

Thus

$$
\begin{aligned}
& \bar{\mu}_{A}(x) \geq \gamma_{\min }\left(\bar{\mu}_{A}\left(x^{*} y\right), \bar{\mu}_{A}(y)\right) . \\
& \text { If } x^{*} y \notin Y \text { and } y \notin Y \text {, then } \\
& \bar{\mu}_{A}(y)=\bar{\mu}_{A}\left(x^{*} y\right)=[0,0] .
\end{aligned}
$$

Thus

$$
\begin{aligned}
& \bar{\mu}_{A}(x) \geq[0,0]=\gamma_{\min }\left(\bar{\mu}_{A}\left(x^{*} y\right), \bar{\mu}_{A}(y)\right) . \\
& \text { If } x^{*} y \in Y \text { and } y \notin Y, \text { then }
\end{aligned}
$$

$$
\bar{\mu}_{A}\left(x^{*} y\right)=\left[\alpha_{1}, \alpha_{2}\right], \bar{\mu}_{A}(y)=[0,0]
$$

Thus

$$
\gamma_{\min }\left(\bar{\mu}_{A}(x * y), \bar{\mu}_{A}(y)\right)=[0,0] \text {. }
$$

Hence

$$
\bar{\mu}_{A}(x) \geq \gamma_{\min }\left(\bar{\mu}_{A}\left(x^{*} y\right), \bar{\mu}_{A}(y)\right)
$$

Similarly for the case $x^{*} y \notin Y$ and $y \in Y$, we get

$$
\bar{\mu}_{A}(x) \geq \gamma_{\min }\left(\bar{\mu}_{A}\left(x^{*} y\right), \bar{\mu}_{A}(y)\right)
$$

On the other hand, since $0 \in Y$, thus $\bar{\mu}_{A}(0)=\left[\alpha_{1}, \alpha_{2}\right]$, that is $\bar{\mu}_{A}(0) \geq \bar{\mu}_{A}(x)$. Hence $\mathrm{A}$ is $\mathrm{i}$-v fuzzy ideal of $\mathrm{S}$.

Theorem 3.5 Let $\mathrm{Y}$ be a nonempty subset of $\mathrm{S}$ and Let $\mathrm{A}$ be an i-v fuzzy set on $\mathrm{S}$ defined by

$$
\bar{\mu}_{A}(x)=\left\{\begin{array}{cc}
{\left[\alpha_{1}, \alpha_{2}\right]} & x \in Y \\
{[0,0]} & x \notin Y
\end{array},\right.
$$

where $\alpha_{1}, \alpha_{2} \in[0,1]$ and $\alpha_{1}<\alpha_{2}$. If $A$ is an i-v fuzzy ideal of $S$, Then $Y$ is an ideal of $S$.

Proof Assume that $A$ is i-v fuzzy ideal of $S$. Then

$$
\bar{\mu}_{A}(0) \geq \bar{\mu}_{A}(x), \bar{\mu}_{A}(x) \geq \gamma_{\min }\left(\bar{\mu}_{A}(x * y), \bar{\mu}_{A}(y)\right)
$$

for all $x, y \in S$. Since $Y$ be nonempty subset of $S$, thus there exists $x \in Y$ such that $\bar{\mu}_{A}(x)=\left[\alpha_{1}, \alpha_{2}\right]$, so

$$
\bar{\mu}_{A}(0) \geq \bar{\mu}_{A}(x)=\left[\alpha_{1}, \alpha_{2}\right] \text {. }
$$

This shows that $0 \in Y$.

If $x^{*} y \in Y$ and $y \in Y$, then

$$
\bar{\mu}_{A}\left(x^{*} y\right)=\bar{\mu}_{A}(x)=\left[\alpha_{1}, \alpha_{2}\right]
$$

Therefore

$$
\begin{aligned}
& \bar{\mu}_{A}(x) \geq \gamma_{\min }\left(\bar{\mu}_{A}\left(x^{*} y\right), \bar{\mu}_{A}(y)\right) \\
& =\gamma_{\min }\left(\left[\alpha_{1}, \alpha_{2}\right],\left[\alpha_{1}, \alpha_{2}\right]\right)=\left[\alpha_{1}, \alpha_{2}\right]
\end{aligned}
$$
$S$.

So $\bar{\mu}_{A}(x)=\left[\alpha_{1}, \alpha_{2}\right]$, that is $x \in Y$. Hence $Y$ is ideal of

Theorem 3.6 If A be an i-v fuzzy ideal of S, then the set

$$
X_{0}=\left\{x \in S \mid \bar{\mu}_{A}(x)=\bar{\mu}_{A}(0)\right\}
$$

is ideal of $\mathrm{S}$. then

Proof It is clear that $0 \in X_{0}$. If $y \in X_{0}$ and $x^{*} y \in X_{0}$, 


$$
\bar{\mu}_{A}\left(x^{*} y\right)=\bar{\mu}_{A}(y)=\bar{\mu}_{A}(0)
$$

Since A be an i-v fuzzy ideal of S, hence

$$
\begin{aligned}
& \bar{\mu}_{A}(0) \geq \bar{\mu}_{A}(x) \geq \gamma_{\text {min }}\left(\bar{\mu}_{A}\left(x^{*} y\right), \bar{\mu}_{A}(y)\right) \\
& =\gamma_{\min }\left(\bar{\mu}_{A}(0), \bar{\mu}_{A}(0)\right)=\bar{\mu}_{A}(0)
\end{aligned}
$$

It follows that $x \in X_{0}$, therefore the set $X_{0}$ is ideal of S.

Let $\bar{\mu}_{A \text { and }} \bar{\mu}_{B}$ be two i-v fuzzy set on $\mathrm{S}$, define the i-v fuzzy set $\bar{\mu}_{\mathrm{A} \cap B \text { on S by }}$

$$
\bar{\mu}_{A \cap B}(x)=\gamma_{\min }\left(\bar{\mu}_{A}(x), \bar{\mu}_{B}(x)\right), x \in S .
$$

Then $\bar{\mu}_{A \cap B}$ is called the intersection of $\bar{\mu}_{A \text { and }} \bar{\mu}_{B}$.

Theorem 3.7 Let A and B be two i-v fuzzy ideals of S. Then the intersection of A and B is also i-v fuzzy ideal of S.

Proof Assume that A and B be two i-v fuzzy ideals of S. Then for all $x, y \in S$, we have

$$
\begin{aligned}
& \bar{\mu}_{A}(0) \geq \bar{\mu}_{A}(x), \bar{\mu}_{A}(x) \geq \gamma_{\min }\left(\bar{\mu}_{A}\left(x^{*} y\right), \bar{\mu}_{A}(y)\right), \\
& \bar{\mu}_{B}(0) \geq \bar{\mu}_{B}(x), \bar{\mu}_{B}(x) \geq \gamma_{\min }\left(\bar{\mu}_{B}\left(x^{*} y\right), \bar{\mu}_{B}(y)\right) .
\end{aligned}
$$

Hence

$$
\begin{aligned}
& \bar{\mu}_{A \cap B}(0)=\gamma_{\min }\left(\bar{\mu}_{A}(0), \bar{\mu}_{B}(0)\right) \\
\geq & \gamma_{\min }\left(\bar{\mu}_{A}(x), \bar{\mu}_{B}(x)\right)=\bar{\mu}_{A \cap B}(x) \\
& \bar{\mu}_{A \cap B}(x)=\gamma_{\min }\left(\bar{\mu}_{A}(x), \bar{\mu}_{B}(x)\right) \\
& \geq \gamma_{\text {min }}\left(\gamma_{\text {min }}\left(\bar{\mu}_{A}\left(x^{*} y\right), \bar{\mu}_{A}(y)\right), \gamma_{\text {min }}\left(\bar{\mu}_{B}\left(x^{*} y\right), \bar{\mu}_{B}(y)\right)\right) \\
= & \gamma_{\min }\left(\gamma_{\text {min }}\left(\bar{\mu}_{A}\left(x^{*} y\right), \bar{\mu}_{B}\left(x^{*} y\right)\right), \gamma_{\min }\left(\bar{\mu}_{A}(y), \bar{\mu}_{B}(y)\right)\right) \\
= & \gamma_{\min }\left(\bar{\mu}_{A \cap B}\left(x^{*} y\right), \bar{\mu}_{A \cap B}(y)\right)
\end{aligned}
$$$$
\text { S. }
$$

Hence the intersection of A and B is also i-v fuzzy ideal of

Theorem 3.8 Assume that $\mathrm{S}$ is a $\mathrm{N}(2,2,0)$ algebra, then $\left(S \times S,{ }^{*}, \Delta,(0,0)\right)$ is also a $\mathrm{N}(2,2,0)$ algebra. Where

$$
\begin{aligned}
& \left(x_{1}, y_{1}\right) *\left(x_{2}, y_{2}\right)=\left(x_{1} * x_{2}, y_{1} * y_{2}\right), \\
& \left(x_{1}, y_{1}\right) \Delta\left(x_{2}, y_{2}\right)=\left(x_{1} \Delta x_{2}, y_{1} \Delta y_{2}\right) \\
& \text { for all }\left(x_{1}, y_{1}\right) \in S \times S \text { and }\left(x_{2}, y_{2}\right) \in S \times S .
\end{aligned}
$$

Proof Assume that $S$ is a $\mathrm{N}(2,2,0)$ algebra, then for all $\left(x_{1}, y_{1}\right) \in S \times S \quad\left(x_{2}, y_{2}\right) \in S \times S$ and $\left(x_{3}, y_{3}\right) \in S \times S$, we have

$$
\begin{aligned}
& \left(x_{1}, y_{1}\right) *\left(\left(x_{2}, y_{2}\right) \Delta\left(x_{3}, y_{3}\right)\right) \\
& =\left(x_{1}, y_{1}\right) *\left(x_{2} \Delta x_{3}, y_{2} \Delta y_{3}\right) \\
& =\left(x_{1} *\left(x_{2} \Delta x_{3}\right), y_{1} *\left(y_{2} \Delta y_{3}\right)\right) \\
& =\left(x_{3} *\left(x_{1} * x_{2}\right), y_{3} *\left(y_{1} * y_{2}\right)\right) \\
& =\left(x_{3}, y_{3}\right) *\left(x_{1} * x_{2}, y_{1} * y_{2}\right) \\
& =\left(x_{3}, y_{3}\right) *\left(\left(x_{1}, y_{1}\right) *\left(x_{2}, y_{2}\right)\right) \\
& \left(\left(x_{1}, y_{1}\right) \Delta\left(x_{2}, y_{2}\right)\right) *\left(x_{3}, y_{3}\right)=y *\left(x^{*} z\right) \\
& =\left(x_{1} \Delta x_{2}, y_{1} \Delta y_{2}\right) *\left(x_{3}, y_{3}\right) \\
& =\left(\left(x_{1} \Delta x_{2}\right) * x_{3},\left(y_{1} \Delta y_{2}\right) * y_{3}\right) \\
& =\left(x_{2} *\left(x_{1} * x_{3}\right), y_{2} *\left(y_{1} * y_{3}\right)\right) \\
& =\left(x_{2}, y_{2}\right) *\left(x_{1} * x_{3}, y_{1} * y_{3}\right) \\
& =\left(x_{2}, y_{2}\right) *\left(\left(x_{1}, y_{1}\right) *\left(x_{3}, y_{3}\right)\right) \\
& (0,0) *\left(x_{1}, y_{1}\right)=\left(0 * x_{1}, 0 * y_{1}\right)=\left(x_{1}, y_{1}\right)
\end{aligned}
$$

Hence by Definition $2.1,\left(S \times S,{ }^{*}, \Delta,(0,0)\right)$ is also a $\mathrm{N}$ $(2,2,0)$ algebra

Let $\mathrm{A}$ and $\mathrm{B}$ be two i-v fuzzy set of $\mathrm{S}$. The direct product of $\mathrm{A}$ and $\mathrm{B}\left(A \times B=\left\{(x, y),\left[\mu_{A \times B}^{L}(x, y), \mu_{A \times B}^{U}(x, y)\right]\right\}\right)$ is define by

$$
\bar{\mu}_{A \times B}(x, y)=\gamma_{\min }\left(\bar{\mu}_{A}(x), \bar{\mu}_{B}(y)\right)
$$

Theorem 3.9 Let $A$ and $B$ be two i-v fuzzy ideals of $S$. Then the direct product $A \times B$ is i-v fuzzy ideal of $S \times S$.

Proof Assume that A and B be two i-v fuzzy ideals of S. Then for all $x, y \in S$, we have

$$
\begin{aligned}
& \bar{\mu}_{A}(0) \geq \bar{\mu}_{A}(x), \bar{\mu}_{A}(x) \geq \gamma_{\min }\left(\bar{\mu}_{A}\left(x^{*} y\right), \bar{\mu}_{A}(y)\right), \\
& \bar{\mu}_{B}(0) \geq \bar{\mu}_{B}(x), \bar{\mu}_{B}(x) \geq \gamma_{\min }\left(\bar{\mu}_{B}\left(x^{*} y\right), \bar{\mu}_{B}(y)\right) .
\end{aligned}
$$

Hence for all $(x, y) \in S \times S$,

$$
\begin{aligned}
& \bar{\mu}_{A \times B}(0,0)=\gamma_{\min }\left(\bar{\mu}_{A}(0), \bar{\mu}_{B}(0)\right) \\
& \geq \gamma_{\min }\left(\bar{\mu}_{A}(x), \bar{\mu}_{B}(y)\right) \\
& =\bar{\mu}_{A \times B}(x, y) .
\end{aligned}
$$

For all $\left(x_{1}, y_{1}\right) \in S \times S$ and $\left(x_{2}, y_{2}\right) \in S \times S$, we have 


$$
\begin{gathered}
\bar{\mu}_{A \times B}\left(x_{1}, y_{1}\right)=\gamma_{\min }\left(\bar{\mu}_{A}\left(x_{1}\right), \bar{\mu}_{B}\left(y_{1}\right)\right) \\
\geq \gamma_{\min }\left(\gamma_{\min }\left(\bar{\mu}_{A}\left(x_{1} * x_{2}\right), \bar{\mu}_{A}\left(x_{2}\right)\right),\right. \\
\left.\gamma_{\text {min }}\left(\bar{\mu}_{B}\left(y_{1} * y_{2}\right), \bar{\mu}_{B}\left(y_{2}\right)\right)\right) \\
=\gamma_{\min }\left(\gamma_{\min }\left(\bar{\mu}_{A}\left(x_{1} * x_{2}\right), \bar{\mu}_{B}\left(y_{1} * y_{2}\right)\right),\right. \\
\left.\gamma_{\min }\left(\bar{\mu}_{A}\left(x_{2}\right), \bar{\mu}_{B}\left(y_{2}\right)\right)\right) \\
=\gamma_{\min }\left(\bar{\mu}_{A \times B}\left(x_{1}^{*} x_{2}, y_{1}^{*} y_{2}\right), \bar{\mu}_{A \times B}\left(x_{2}, y_{2}\right)\right) \\
=\gamma_{\min }\left(\bar{\mu}_{A \times B}\left(\left(x_{1}, y_{1}\right) *\left(x_{2}, y_{2}\right)\right), \bar{\mu}_{A \times B}\left(x_{2}, y_{2}\right)\right)
\end{gathered}
$$

Therefore the direct product $A \times B$ is i-v fuzzy ideal of $S \times S$.

Theorem 3.10 Let $f_{\text {be a surjective homomorphism from }}$ $\mathrm{N}(2,2,0)$ algebra $S_{1}$ to $S_{2}, f(0)=0$. If $\bar{v}$ is an i-v fuzzy ideal of $S_{2}$, then $f^{-1}(\bar{v})$ is i-v fuzzy ideal of $S_{1}$. Where

$$
f^{-1}(\bar{v})(x)=\bar{v}(f(x)), x \in S_{1} .
$$
then

Proof For all $x, y \in S_{1}$, since $\bar{v}$ is i-v fuzzy ideal of $S_{2}$,

$$
\begin{aligned}
f^{-1}(\bar{v})(0)=\bar{v}(f(0))=\bar{v}(0) \geq \bar{v}(f(x))=f^{-1}(\bar{v})(x) \\
\quad f^{-1}(\bar{v})(x)=\bar{v}(f(x)) \\
\quad \geq \gamma_{\min }\left(\bar{v}\left(f(x)^{*} f(y)\right), \bar{v}(f(y))\right) \\
=\gamma_{\min }\left(\bar{v}\left(f\left(x^{*} y\right)\right), \bar{v}(f(y))\right) \\
=\gamma_{\min }\left(f^{-1}(\bar{v})\left(x^{*} y\right), f^{-1}(\bar{v})(y)\right)
\end{aligned}
$$

Hence $f^{-1}(\bar{v})$ is i-v fuzzy ideal of $S_{1}$.

Theorem 3.10 Let $f_{\text {be a surjective homomorphism from }}$ $\mathrm{N}(2,2,0)$ algebra $S_{1}$ to $S_{2}, f(0)=0$. If $\bar{\mu}$ is an i-v fuzzy ideal of $S_{1}$, then $f(\bar{\mu})$ is i-v fuzzy ideal of $S_{2}$. Where

$$
f(\bar{\mu})(y)=\gamma_{\max }\{\bar{\mu}(x) \mid f(x)=y\}
$$

Definition 3.11 If $\bar{\mu}$ is an i-v fuzzy set of $S_{\text {and Let }} f_{\text {is }}$ a mapping defined on $S$, the i-v fuzzy set $\bar{\mu}_{\text {in }}^{f} f(S)$ defined by

$$
\bar{\mu}^{f}(y)=\gamma_{\max }\left\{\bar{\mu}(x) \mid x \in f^{-1}(y), x \in S\right\}
$$

is called the image of $\bar{\mu}_{\text {under }} f$.
Theorem 3.12 Let $S_{1}$ and $S_{2}$ be two N(2,2,0) algebra and $f_{\text {be a epimorphism from }} S_{1}$ to $S_{2}$. If $\bar{\mu}$ is an i-v fuzzy ideal of $S_{1}$, then $\bar{\mu}^{f}$ is an i-v fuzzy ideal of $S_{2}$.

\section{REFERENCE}

[1] Deng Fangan,Xu Yang. On N(2,2,0) algebras. Journal of southwest jiaotong university, 1996, vol 31, pp 457-463.

[2] Deng Fangan,Xu Yang and Yuan Jian. Ideal and relevant ideal of $\mathrm{N}(2,2,0)$ algebra . Journal of Hanzhong Teachers College, 1998, 16(1): 6-9.

[3] Li Xudong. Image and converse image of translation transform of $\mathrm{N}(2,2,0)$ algebra. Journal of Mathematical Research and Exposition, 2005,25(1):148-153.

[4] Li Xudong. Two classes congruence decomposition on $\mathrm{N}(2,2,0)$ algebras . Journal of Lanzhou University(Natural Science), 2005, 41(5): $120-122$.

[5] Li Xudong, Song xuemei. Another congruence decomposition on $\mathrm{N}(2,2,0)$ algebras.Journal of Northwest Normal University(Natural Science),2010,46(6): 23-29.

[6] Deng Fangan. RC-semigroups of N $(2,2,0)$ algebra. Journal of Shandong University(Natural Science), 2011,46(1):8-11.

[7] Chen Lu. Medial idempotents of $(2,2,0)$ algebra.Pure and Applied Mathematics,2011,27(4):433-436.

[8] Deng Fangan, Yong Longquan. Regular semigroups of N $(2,2,0)$ algebra. Advances in Mathematics,2012,41(6):665-671.

[9] Zadeh L A. Fuzzy sets. Information and Control, 1965(8):338-353.

[10] Rosenfeld. A. Fuzzy groups. Journal of Mathematical Analysis and Applications, 1971(35):512-517.

[11] Wang Fengxiao. On $(\lambda, \mu)$-fuzzy subalgebras in Boolean algebras.

Applied Mathematics A Journal of Chinese Universities (Ser.A), 2011, vol 26, pp495-500. 